\title{
Dural sac localization using myelography and its application to the lumbosacral epidural in dogs
}

\author{
Ana Zapata ${ }^{1 *}$, Carlos Ros², Elena Ríos Álvarez ${ }^{1}$, Myriam Martín ${ }^{1}$ and Alejandra García De Carellán Mateo ${ }^{2}$ \\ ${ }^{1}$ Veterinary Hospital of Catholic University of Valencia San Vicente Mártir, Valencia, Spain \\ ${ }^{2}$ Department of Animal Medicine and Surgery, Faculty of Veterinary Sciences, Catholic University of Valencia \\ "San Vicente Mártir", Valencia, Spain
}

\begin{abstract}
Background: The techniques described for the identification of the lumbosacral (LS) epidural space in dogs do not guarantee the needle position or an accidental subarachnoid puncture, especially in small size dogs.

Aim: To determine the relationship between body weight and the location of the dural sac (DS) using myelography in dogs, and to determine the possibility of subarachnoid puncture during LS epidural based on the position of the DS.

Methods: Four masked observers evaluated 70 myelographic studies of dogs, annotating the vertebrae where the DS ended, if it was localized before or after the LS space, and if accidental subarachnoid puncture during LS epidural injection was possible (yes/no). Body weight ( $\mathrm{kg}$ ) was categorized into: less than $10 \mathrm{~kg}$, between 10 and $20 \mathrm{~kg}$, and more than $20 \mathrm{~kg}$ and was also converted to body surface area (BSA) as a continuous variable.

Results: The DS ended at the LS space or caudally in 50\% of dogs. There was a statistically significant difference between the position of the DS and the dog's BSA $(p=0.001)$. The DS ended caudal to the LS space in $72.7 \%$ of dogs weighing $<10 \mathrm{~kg}$, in $25 \%$ of dogs between 10 and $20 \mathrm{~kg}$ and in $15 \%$ of dogs in the $>20 \mathrm{~kg}$ category. The observers considered a possible subarachnoid puncture during LS epidural in $69.7 \%$ of patients $<10 \mathrm{~kg}, 16.6 \%$ on those between 10 and $20 \mathrm{~kg}$, and in $11.7 \%$ of the dogs $>20 \mathrm{~kg}$.

Conclusion: The DS ended caudal to the LS space in almost $3 / 4$ dogs in the $<10 \mathrm{~kg}$ category, so accidental subarachnoid puncture during LS epidural is highly possible in this weight range.

Keywords: Body weight, Dog, Dural sac, Lumbosacral epidural, Myelography.

\section{Introduction}

Epidural drug administration is a loco-regional technique broadly used in veterinary anaesthesia because is relatively easy to perform with a low rate of complications (Campoy et al., 2015; Garcia-Pereira, 2018). It can decrease the adverse effects of general anaesthetics by reducing their dose requirements as well as intraoperative and postoperative opioid consumption for thoracic, abdominal, hind limb, and perineal surgeries (Garcia-Pereira, 2018). The most commonly used drugs for epidural administration in small animals are local anaesthetics either alone or mixed with other analgesics, such as opioids, alpha-2 agonists, or ketamine to provide multimodal analgesia (DukeNovakovski, 2016). The epidural space is a virtual space within the spinal canal between the duramater and the periosteum, occupied by nerve roots, adipose tissue, and venous plexus (Jones, 2001). In dogs, the lumbosacral (LS) space is generally the preferred site of injection for epidural drug administration due to the easy identification of anatomical landmarks and low risk of spinal cord damage (Duke-Novakovski, 2016). The epidural injection can be performed with the patient in lateral or sternal recumbence, normally with the pelvic

limbs extended in forward direction. The iliac crests, the spinous process of the last lumbar vertebra, and the first sacral vertebra, are the anatomical landmarks to identify the LS space. The needle is carefully advanced through the subcutaneous tissue, muscle layers, and ligamentum flavum into the vertebral canal (DukeNovakovski, 2016). The techniques described for correct identification of the epidural space include pop sensation, hanging drop, loss of resistance, modified Baraka technique, radiography, contrast radiography, nerve stimulation, and ultrasound guidance (GarciaPereira, 2018), but they do not guarantee the position of the needle or the avoidance of the subarachnoid space, possibly leading to an unnoticed intrathecal or spinal injection instead of epidural technique (Otero et al., 2014; Adami and Gendron, 2017).

The veterinary literature describes that the spinal cord ends approximately at the 6th or 7th lumbar vertebrae in dogs (Campoy et al., 2015; Otero and Portela, 2017). The spinal cord terminates forming the Conus medullaris (CM) (Garosi, 2012) surrounded by cerebrospinal fluid (CSF) within the dural sac (DS). The DS is continued by a filament, which attaches it to a sacral or a caudal vertebra (Fletcher, 2013). Some
\end{abstract}


veterinary studies showed a relationship between the size of the dog and the spinal cord termination in the CM, extending approximately to the L6 vertebral body in most large breed dogs and the L7 vertebral body in small breed dogs (Morgan et al., 1987). However, there is controversy in the veterinary literature regarding the location of the DS in dogs.

The aims of this study were to describe the position of the DS using myelography in dogs and determine if there is a relationship between the body weight and the location of the DS. The second aim is to study the risk of subarachnoid puncture during LS epidural in dogs, based on the position of the DS. Our hypothesis is that in small breed dogs the DS ending will reach the first sacral vertebra, thus increasing the risk of accidental puncture of the subarachnoid space during the LS epidural.

\section{Materials and Methods}

\section{Animals' criteria}

The study design was a retrospective cohort study including myelographies of canine patients with acute onset of spinal cord dysfunction. The medical records of Veterinary Hospital of Catholic University of Valencia San Vicente Mártir were reviewed and all canine myelographies between December 2015 and May 2019 were selected. Exclusion criteria were incomplete patient records, cases on which contrast radiographies did not include the DS or if the vertebra where the DS ended was not completely visualized on the radiographs.

\section{Clinical information}

The following parameters were recorded from patient's medical records: gender, body weight $(\mathrm{kg})$, age and breed, number of lumbar vertebrae, location of the lumbar puncture, and the presence or absence of spinal cord compression. To facilitate statistical analyses, body weight was converted to body surface area (BSA) using the following formula: BSA $\left(\mathrm{m}^{2}\right)=0.101 \times$ [(body weight in $\mathrm{kg})^{2 / 3}$ ] (Plumb, 2011). In addition, dogs were classified according to their body weight in three different groups: less than $10 \mathrm{~kg}$, between 10 and $20 \mathrm{~kg}$, and more than $20 \mathrm{~kg}$. Dogs were also categorized into brachycephalic or non-brachycephalic breed.

Myelography protocol

All myelographies were carried out in the same manner: pre and postcontrast lateral and ventrodorsal radiographs (indirect digital radiographs, Toshiba Rotanode ${ }^{\mathrm{TM}}$ E7239X, Japan) of all spinal cord segments were acquired under general anaesthesia. For the lumbar puncture, patients were positioned in right lateral recumbence with the pelvic limbs in a neutral position, perpendicular to the vertebral column. Lumbar puncture was performed percutaneously using a Quincke spinal needle (Becton Dickison S.A, Madrid, Spain or Spinocan B. Braun Melsugen AG, Melsugen, Germany) $22 \mathrm{G} 0.7 \times 63 \mathrm{~mm} / 0.7 \times 38 \mathrm{~mm}$ or $0.7 \times 75$ $\mathrm{mm} / 0.7 \times 40 \mathrm{~mm}$ according to the size of the patient.
In order to confirm the correct position of the needle within the subarachnoid space, CSF flow through the hub of the needle was observed. The myelography of all spinal cord segments was performed using iodinated contrast media (OMNIPAQUE ${ }^{\mathrm{TM}} 300 \mathrm{mg}$ iodine $/ \mathrm{ml}$, GE Healthcare Bio-Sciences, S.A.U., Madrid, Spain). The contrast was manually injected for complete filling of the subarachnoid space up to $\mathrm{C} 1$ or a maximum volume of $0.45 \mathrm{ml} / \mathrm{kg}$.

All myelographic studies were evaluated with commercially available DICOM digital image viewing software (Aycan, workstation v3.6). Only lateral radiographs could be evaluated, due to superposition with pelvic structures in the ventro-dorsal views. Four observers blinded to patient's medical record information reviewed the studies that met the inclusion criteria. The observers annotated the vertebra where the DS ended (L6, L7/L8, or S1), and this vertebra was selected as a reference vertebra (RV) (Fig. 1A and B). The RV was measured longitudinally (at the level of the middle part of the vertebral body) in $\mathrm{mm}$ (Line a in Fig. 1C and D). The observers also measured the DS, and a percentage with respect to the RV measurement was calculated $(\%)$. To perform the measurements of

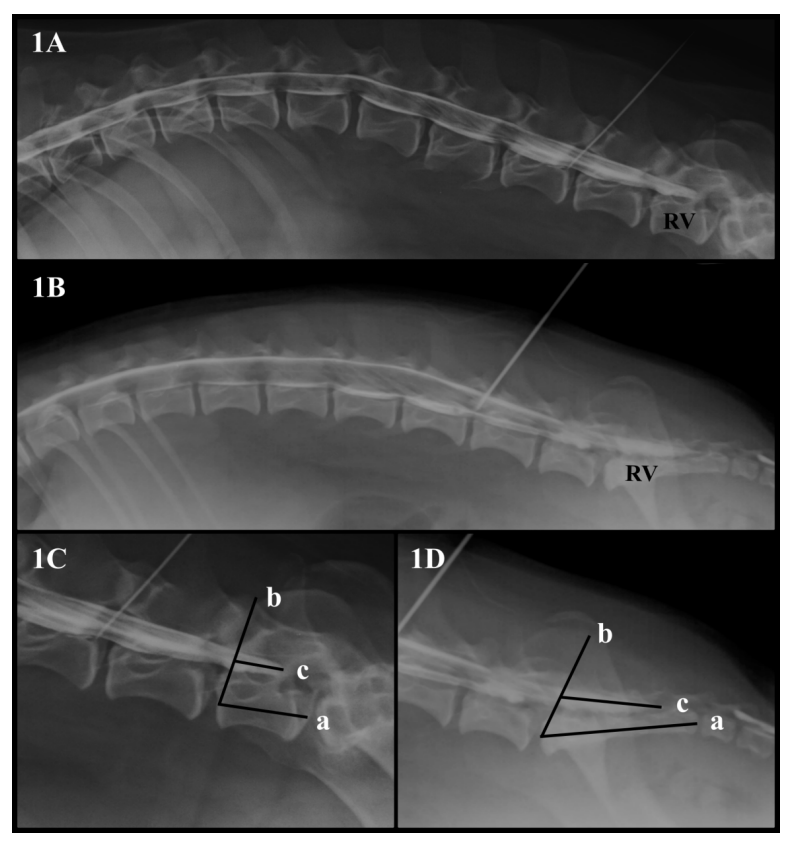

Fig. 1. (A). Canine lateral myelographic image where DS ends before the LS space. (B) Lateral myelographic image where the DS ends after LS space. (C) Measurement method used for the RV and DS ending where L7 was considered the $R V$. Line $a=R V$ measurement. Line $b=$ starting point for DS measurement. Line $\mathrm{c}=$ DS measurement. (D) Measurement method used for RV and the DS ending where the sacrum was considered the RV. Line $a=R V$ measurement, Line $\mathrm{b}=$ starting point for the DS measurement. Line $\mathrm{c}=\mathrm{DS}$ measurement. 
the DS, a starting point was set drawing a line from the rostral midpoint epiphysis of the vertebral body of the RV to the caudal articular facet of the vertebra immediately rostral to it (Line b in Fig. 1C and D). The direction of the parallel line mimicked the insertion of a Tuohy needle during an epidural injection when L7 was considered the RV. From this line, the DS was measured in $\mathrm{mm}$ drawing a line parallel to the floor of the vertebral canal (Line $\mathrm{c}$ in Fig. 1C and D).

Lastly, the LS space was identified as the intervertebral disc space between the last lumbar vertebrae and the first sacral vertebrae. The observers were asked to assess the possibility of accidental subarachnoid puncture during LS epidural injection (yes/no), based on where the DS ended: before the LS space, at the LS space or after the LS space.

\section{Statistical analysis}

The data obtained were analysed using a commercially available statistical software [Statistical Package for the Social Sciences (SPSS) for Mac, version 20, IBM SPSS Inc, Madrid, Spain]. Categorical variables were summarized in frequencies. The distribution of normality was evaluated by the Shapiro-Wilk test and the observation of histograms. Parametric data was summarized in mean and standard deviation and non-parametric data in median and interquartile range (IQR).

The Cohen's Kappa was used to measure the interobserver agreement in RV (L6/L7/L8/S1) position and possibility of accidental subarachnoid puncture during LS epidural injection (yes/no). The inter-rater reliability for the RV and DS measurements was calculated using the intra-class correlation coefficient.

Fisher's Exact test was used to compare the RV where the DS ended with the weight category of the dogs. Chisquare test was used to compare the brachiocephalic breed with the possibility of accidental subarachnoid puncture during LS epidural. The Mann-Whitney U test was used to compare the possibility of accidental subarachnoid puncture during the LS epidural with the median BSA of the dogs. Multiple logistic regressions were used to study the factors that could influence the DS position. Binary logistic regression was used to test how the BSA affected the probability of accidental subarachnoid puncture during the LS epidural and odds ratios (OR) and confidence intervals $(95 \% \mathrm{CI})$ were calculated. Statistical significance was considered if $p<$ 0.05 and a moderate agreement in coefficients $0.4-0.6$, high in $0.6-0.8$ and almost perfect $>0.8$.

\section{Ethical approval}

The Project was approved by the Catholic University of Valencia San Vicente el Mártir Ethics Committee UCV/2018-2019/103.

\section{Results}

\section{Study population}

Ninety-two myelographies were identified, of which 22 had to be excluded from the study because the end of the DS or RV could not be measured due to incomplete appearance in the radiographic images. Seventy myelographies were finally included in the study. Gender distribution was 48 males and 22 females, with an average age of 7 years (range and IQR 1.5-15; 5.3 years), a median body weight of $10.7 \mathrm{~kg}$ (range and IQR, 2.3-72; $10.6 \mathrm{~kg}$ ) and a BSA of $0.492 \mathrm{~m}^{2}$ (range and IQR, $0.176-1.748 ; 1.572 \mathrm{~m}^{2}$ ). Body weight distribution was: $47.1 \%$ of the cases $(n=33)$ were included in the less than $10 \mathrm{~kg}$ category, $34.3 \%$ of the cases $(n=24)$ were between 10 and $20 \mathrm{~kg}$ and $18.6 \%$ of the cases $(n$ $=13$ ) were more than $20 \mathrm{~kg}$. Breeds included were: Cross-breed dogs $(n=17)$, French Bulldog $(n=10)$, Yorkshire Terrier $(n=9)$, Maltese $(n=4)$, Pug $(n=4)$, Beagle $(n=3)$, Cocker Spaniel $(n=3)$, Chihuahua $(n$ $=3)$, Dachshund $(n=3)$, Pinscher Toy $(n=3)$, Great Dane $(n=2)$, American Staffordshire Terrier $(n=1)$, Cotton de Tulear $(n=1)$, Dalmatian $(n=1)$, Doberman $(n=1)$, Dogue de Bordeaux $(n=1)$, West Highland White Terrier $(n=1)$, Ibizan hound $(n=1)$, Weimaraner $(n=1)$, and Miniature Schnauzer $(n=1)$.

Myelography of the studied dogs based on breed and their body weight

Of the seventy myelographies evaluated, 66 dogs had 7 lumbar vertebrae, of which, one French Bulldog and one Pug had L1 as a transitional vertebra (vertebrae that have characteristics of two different anatomic divisions) and four $\operatorname{dogs}$ (Pug $n=2$, Yorkshire Terrier $n=2$ ) had eight lumbar vertebrae. Vertebral canal puncture was performed at L4-L5 $(n=13), \mathrm{L} 5-\mathrm{L} 6(n$ $=53)$, or L6-L7 $(n=4)$ interarcuate foramen. Of the four patients who underwent puncture L6-L7, three of them had eight lumbar vertebrae. Forty-eight patients showed a compressive extradural myelographic pattern, six patients had minimally compressive extradural myelographic pattern consistent with presumptive acute non-compressive nucleous pulposus extrusion, nine patients had intramedullary pattern and seven patients had normal myelography.

The observers identified that the DS ended before the LS space in $50 \%$ of dogs $(n=35)$. The RV where the DS ended in the different weight groups is presented in Table 1.

The likelihood of the association of DS puncture with the bodyweight of the studied dogs

Within the weight categories, the observers considered that the accidental subarachnoid puncture during LS epidural was possible in $69.7 \%$ (25/33) of dogs weighing less than $10 \mathrm{~kg}, 16.6 \%$ on those between 10 and $20 \mathrm{~kg}$ $(8 / 24)$ and in $11.7 \%$ of the dogs that weighted more than $20 \mathrm{~kg}(2 / 13)$. There was no statistical difference between the possibility of accidental subarachnoid puncture during LS epidural in brachiocephalic versus non-brachiocephalic breed dogs.

Cohen's Kappa indicated that the agreement between observers regarding the RV position and for the possibility of accidental subarachnoid puncture during LS epidural was high: $0.644-0.765(p=0.001)$ and 
Table 1. Summary of the RV and position of the dural sac identified by myelography in dogs and its relation to body weight category.

\begin{tabular}{lccc}
\hline RV and DS location in 70 dogs & L6 & L7/L8 & S1 \\
\hline Dogs weighing less than $10 \mathrm{~kg}(n=33)$ & 0 & $9(27.3 \%)$ & $24(72.7 \%)$ \\
Dogs weighing between 10 and $20 \mathrm{~kg}(n=24)$ & $6(25.0 \%)$ & $12(50 \%)$ & $6(25.0 \%)$ \\
\hline Dogs weighing more than $20 \mathrm{~kg}(n=13)$ & $2(15.4 \%)$ & $9(69.2 \%)$ & $2(15.4 \%)$ \\
\hline
\end{tabular}

(RV): Reference Vertebra; (DS): Dural Sac; (L6): Sixth Lumbar Vertebra; (L7): Seventh Lumbar Vertebra; (L8): Eighth Lumbar Vertebra; (S1): First Sacral Vertebra.

$0.600-0.743(p=0.001)$, respectively. The intra-class correlation coefficients indicated that the inter-rater reliability was almost perfect for the RV measurements $0.812(p=0.001)$ and moderate for DS measurements $0.571(p=0.001)$.

The Fisher's Exact test indicated that there was a statistically significant difference between the RV and the weight category of the dogs $(p=0.001)$. The MannWhitney $U$ test indicated that there was a statistically significant difference between the position of the DS and the median $\operatorname{BSA}(p=0.001)$.

The multivariate logistic regression indicated that the two variables that influenced significantly the possibility of accidental DS puncture during LS epidural were the weight category $(p=0.046)$ and the BSA $(p=0.001)$. Using the binary logistic regression, BSA had a negative impact on the probability of DS puncture during LS epidural; therefore the smaller the BSA of the dog, the higher the probability $(\mathrm{OR}=0.002$, 95\% CI 0.00-0.068).

\section{Discussion}

The findings obtained in our study showed that in 50\% of the dogs the DS reached the LS space or extended more caudally. The DS ended caudal to the LS space in $72.7 \%$ of patients in the less than $10 \mathrm{~kg}$ category, so accidental subarachnoid puncture during LS epidural would be possible in this body weight range. In contrast, the DS only reached the sacrum in $25 \%$ and $15.4 \%$ of dogs in the body weight categories of $10-20 \mathrm{~kg}$ and more than $20 \mathrm{~kg}$, respectively. A statistically significant difference was observed between the BSA of the dogs and the location of the DS, decreasing the probability of subarachnoid puncture during the LS epidural as BSA increased. These findings coincide with reported complications of the epidural technique in dogs, where it is described that there is a greater probability of inadvertent spinal puncture in small breed dogs since the spinal cord extends more caudally than in large breed dogs (Campoy et al., 2015).

In the veterinary studies where the $\mathrm{CM}$ was measured, it was observed that the $\mathrm{CM}$ ended in the caudal half of L6 and the cranial part of L7, extending more caudally in small breed dogs (Fletcher and Kitchell, 1966). By means of cisternal myelography, Morgan et al. (1987) observed that the intumescence of the spinal cord was located more caudally in Dachshunds (L6-L7) than in German Shepherd dogs (L4). But it is not entirely correct to apply these findings to the possibility of performing an inadvertent spinal puncture during LS epidural, since the DS is caudally located to the CM in dogs and anchored to the sacrum by the Fillum terminale (Fletcher, 2013).

Although there is a relationship between body weight and the end of the CM in dogs, there is controversy regarding the position of the DS in the veterinary literature. The results of our study showed that only in $50 \%$ of dogs the DS reached the LS space, data that do not match with previous literature (Lang, 1988; Pogorevc et al., 2016). By means of myelography, Pogorevc et al. (2016) showed that the DS reached the LS space or extended caudal to it in $80 \%-91 \%$ of German Shepherds and Belgian Mallinois. In an anatomical study (Marín-García et al., 1995), using 10 German Shepherds the authors found that the DS reached the sacrum, however they used a very small number of patients and they did not compare with small breed dogs.

Recently, it has been described with the use of magnetic resonance imaging (MRI) that the DS remained in a constant position regardless of the size of the dog. It was observed that in most of the patients, the DS extended to the LS space or caudal to it (Khan et al., 2019). In contrast, another study where MRI was used to compare Cavalier King Charles Spaniels (CKCS) with other small breed dogs up to $14 \mathrm{~kg}$ of body weight, showed that only in $66 \%$ of CKCS the DS reached the LS space, data more similar to our study (Sparks et al., 2019).

This controversy in the findings of different publications may be due to pathologies associated with breeds, as hypothesised by Sparks et al. (2019) where the thoracolumbar presence of syringomyelia was associated with a more caudal termination of the DS in CKCS. Additionally, results published by MarínGarcía et al. (1995) and Pogorevc et al. (2016), in which the DS extended to the LS or caudally in most of their patients could be breed associated, as the population studied were working large breed dogs with cauda equina syndrome or LS stenosis. In our study, the DS did not extend more caudally in brachycephalic dogs compared with other dogs. The individual breeds 
could not be included in the statistical analysis due to the heterogenicity of our data, and therefore we could not rule out this hypothesis.

Using MRI, the end of the CM can be evaluated, however, an erroneous perception of the location of the DS can be made and a specific sequence is required to differentiate fat (epidural) from liquid (CSF). Those sequences include the "fat suppression or balanced short tau inversion recovery and steady-state free precession sequences (SSFP)" (Reina et al., 2007; Ricciardi, 2018). Another possible source of error in the studies using myelography is that the injection speed and volume used during myelography could have expanded the DS and extended it more caudally, giving a greater perception of the risk of puncture during epidural LS in our study.

The usual techniques described for the correct identification of the epidural space do not guarantee the needle position, and therefore an inadvertent intrathecal injection may be possible (Otero et al., 2014). Although there are several publications regarding complications associated with epidural injection in dogs (Jones, 2001; Martorell and Cilli, 2020), none described spinal cord damage during the performance of an epidural technique. In contrast, a microscopic examination of the spinal cord after myelography performed at L5-L6 intervertebral space, revealed haemorrhage, axonal degeneration, and gliosis in five dogs, showing the direct trauma after spinal cord puncture (Kishimoto et al., 2004). After these findings, some authors have recommended the LS space as an alternative to the conventional lumbar myelography puncture site (L5-L6) in dogs, since it often allows a subarachnoid puncture for the injection of contrast medium avoiding damage to the spinal cord (Iseri et al., 2004).

One important limitation of our study is that due to the retrospective design the risk of subarachnoid puncture during epidural injection was subjectively evaluated by the observers. Future prospective studies for correct identification of the needle position during LS epidural in different size dogs are needed to confirm our hypothesis.

Other limitations of our study are the low number of patients weighing more than $20 \mathrm{~kg}$, since they only represented $18.6 \%$ of the population. Additionally, our population included animals with spinal pathology, some of them with spinal cord compression and we do not know how this may have influenced the DS position. The position of the patient also might have influenced the location of the DS, since the sternal position with the head raised 30 degrees and extremities extended cranially, significantly increases the distance between spinous process of L6-L7 and the LS angle in dogs (Concetto et al., 2012). However, Lang (1988) showed that during cisternal myelography the manipulation of the spine in flexion and extreme extension does not change the shape or position of the DS. Likewise, future studies comparing our results with other techniques such as computed tomography and MRI with SSFP could provide more accurate data on the location of DS in canine patients.

In our study, the risk of accidental DS puncture should be taken into account when performing a LS epidural in dogs weighing less than $10 \mathrm{~kg}$. Likewise, future studies comparing our results with other diagnostic imaging techniques such as computed tomography and MRI could provide more accurate data on the location of DS in canine patients.

\section{Conflict of interest}

The authors declare that there is no conflict of interest. Author's contributions

All authors discussed the results and contributed to the final preparation of the manuscript. AZ: Study design, data collection, and interpretation. Writing and preparation of the manuscript. CR: Study design, data management as blinded observer. Data interpretation. Preparation and revision of the manuscript. ERA: Data management as blinded observer. Revision of the manuscript. MM: Data management as blinded observer. Revision of the manuscript. AGdCM: Study design, data management as blinded observer. Statistical analysis and data interpretation. Preparation and revision of the manuscript.

\section{References}

Adami, C. and Gendron, K. 2017. What is the evidence? The issue of verifying correct needle position during epidural anaesthesia in dogs. Vet. Anaesth. Analg. 44, 212-218.

Campoy, L., Read, M. and, Peralta, S. 2015. Canine and feline local anesthetic and analgesic techniques. In Lumb and Jones Veterinary Anesthesia and Analgesia, 5th ed., Wiley-Blackwell, Eds., Thurmon, J.C., William, J.T., Grimm, K.A., Lumb, V.L. and Jones, E.W. Iowa, IA, pp: 847-851.

Concetto, S.D., Mandsager, R.E., Riebold, T.W., Stiger-Vanegas, S.M. and Killos, M. 2012. Effect of hind limb position on the craniocaudal length of the lumbosacral space in anesthetized dogs. Vet. Anaesth. Analg. 39, 99-105.

Duke -Novakovski, T. 2016. Pain management II: local and regional anaesthetic techniques. In BSAVA manual of canine and feline anaesthesia and analgesia, 3rd ed., Small Animal Veterinary Association, Eds., Duke Novakovski, T., Vries, M. and Seymour, C. Gloucester, UK, pp: 152-155.

Fletcher, T.F. 2013. Spinal cord and meninges. In Miller's anatomy of the dog, 4th ed. Eds., Evans, H.E., Miller, M.E. and De Lahunta, A. St. Louis, MO: Elsevier Saunders, pp: 59-610. 
Fletcher, T.F. and, Kitchell, R.L. 1966. Anatomical studies on the spinal cord segments of the dog. Am. J. Vet. Res. 27, 1759-1767.

Garcia-Pereira, F. 2018. Epidural anesthesia and analgesia in small animal practice: an update. Vet. J. 242, 24-32.

Garosi , L. 2012. Lesion localization and differencial diagnosis. In BSAVA manual of canine and feline neurology, 4th ed., Small Animal Veterinary Association, Platt, S.R. and Olby, N.J. Gloucester, UK, pp: 28-29.

Iseri, T., Yamada, K. and Ueno, H. 2004. Lumbosacral myelography in dogs -a safer technique. J. Vet. Med. Sci. 66, 71-72.

Jones, R.S. 2001. Epidural analgesia in the dog and cat. Vet. J. 161, 123-131.

Khan, Z., Munro, E., Shaew, D. and Faller, K.M. 2019. Variation in the position of the conus medullaria and dural sac in adult dogs. Vet. Rec. 185, 20.

Kishimoto, M., Yamada, K., Ueno, H., Kobayashi, Y. and Wisner, E. 2004. Spinal cord effects from lumbar myelographic injection technique in the dog. J. Vet. Med. Sci. 66, 67-69.

Lang, J. 1988. Flexion-extension myelography of the canine cauda equina. Vet. Radiol. Ultrasound. 29, 242-257.

Marín-García, P., González-Soriano, J., MartinezSainz, P., Contreras-Rodríguez, J., Corral-Gros, C. and Rodríguez-Veiga, E. 1995. Spinal cord central canal of the German shepherd dog: Morphological, histological, and ultrastructural considerations. J. Morphol. 224, 205-212.

Martorell, M.M. and Cilli, F. 2020. Cardiopulmonary arrest after epidural injection followed by successful resuscitation using an anticholinergic drug. Vet. Anaesth. Analg. S1467-2987, 30192-30196.

Morgan, J.P., Atiola, M. and Bailey, C.S. 1987. Vertebral canal and spinal cord mesuration: a comparative study of its effects on lumbosacral myelography in the Dachshund and German Shepherd dog. J. Am. Vet. Med. Assoc. 191, 951-957.

Otero, P.E. and Portela, D.A. 2017. Abordaje del espacio epidural. In Manual de anestesia regional en animales de compañía: Anatomía para bloqueos guiados por ecografía y neuroestimulación, Eds., Otero, P.E. and Portela, D.A. Buenos Aires, Argentina: Ciudad Autónoma de Buenos Aires, pp: 273-275.

Otero, P.E., Verdier, N., Ceballos, M.R., Tarragona, L., Flores, M. and Portela, D.A. 2014. The use of electrical stimulation to guide epidural and intrathecal advancement at the L5-L6 intervetrebral space in dogs. Vet. Anaesth. Analg. 41, 543-547.

Plumb, D.C. 2011. Plumb's veterinary drug handbook, 7th ed. Stockholm, Sweden: Wiley Blackwell.

Pogorevc, E., Lukanc, B., Seliškar, A., Pelc, R. and Zorko, B. 2016. Radiological comparision of lumbosacral anatomy between German and Belgian Sherherd (malinois) working dogs. Slov. Vet. Res. 53, 219-227.

Reina, M.A., Pulido, P., Casedo, J., Villanueva, M.C., López, A., De Andrés, J.A. and Sola, R.G. 2007. La grasa epidural en diferentes patologías. Aportaciones de la resonancia magnética y posibles implicaciones en la anestesia neuroaxial. Rev. Esp. Anestesiol. Reanim. 54, 173-183.

Ricciardi, M. 2018. Principles and applications of the balanced steady-state free precession sequence in small animal low-field MRI. Vet. Res. Commun. 42, 65-86.

Sparks, C.R., Robertson, I. and Olby, N.J. 2019. Morphometric analysis of spinal cord termination in Cavalier King Charles Spaniels. J. Vet. Intern. Med. 33, 717-725. 